\title{
Correction to "An Efficient Game Form for Multi-Rate Multicast Service Provisioning"
}

\author{
Ali Kakhbod and Demosthenis Teneketzis Fellow, IEEE
}

$\mathbf{T}$ HIS note corrects an error in our paper, "An Efficient Game Form for Multi-Rate Multicast Service Provisioning" (reference [1]), which presents a rate allocation mechanism for multi-rate multicast service provisioning in networks with arbitrary topology and strategic users. The mechanism presented in [1] includes a tax function which is not differentiable with respect to the rate allocations. To obtain a Nash implementation, however, we need a tax function that is differentiable with respect to these allocations. We correct this error as follows.

We consider the problem formulated in [1]. We use the same notation as in [1]. To help the reader, we repeat here the notation used in this note.

$$
\begin{aligned}
& G_{i}:=\text { Multicast group } i . \\
& \mathcal{N}:=\left\{G_{1}, G_{2}, \cdots, G_{N}\right\} . \\
&\left(j, G_{i}\right):=\text { User } j \text { in multicast group } i . \\
& \mathcal{R}_{\left(j, G_{i}\right)}:=\text { Route of user }\left(j, G_{i}\right) . \\
& \Xi_{\left(j, G_{i}\right)}^{l}:=\text { The term that appears in the tax function } t_{\left(j, G_{i}\right)}^{l} \\
& \quad \text { and is expressed in terms of indicator functions. } \\
& G_{i}^{\max (l):=} \text { Set of users in } G_{i} \text { that request the maximum } \\
& \quad \text { amount of bandwidth at link } l \text { among all } \\
& \quad \text { users in } G_{i} . \\
& Q_{l}:=\text { Set of multicast groups that use link } l . \\
& x_{G_{i}}(l):=\text { Bandwidth requested by } G_{i} \text { in link } l .
\end{aligned}
$$

\section{Specification of the game form/mechanism:}

Message space: The message space is the same as that of the mechanism presented in [1]. A message of user $\left(j, G_{i}\right), j \in$ $G_{i}, G_{i} \in \mathcal{N}$ is of the form

$$
m_{\left(j, G_{i}\right)}=\left(x_{\left(j, G_{i}\right)}, \pi_{\left(j, G_{i}\right)}^{l_{j_{1}}}, \pi_{\left(j, G_{i}\right)}^{l_{j_{2}}}, \cdots, \pi_{\left(j, G_{i}\right)}^{l_{i_{\mid \mathcal{R}}\left(j, G_{i}\right) \mid}}\right),
$$

where $x_{\left(j, G_{i}\right)}$ denotes the (non-negative) bandwidth user $\left(j, G_{i}\right)$ requests at all the links of his route $\mathcal{R}_{\left(j, G_{i}\right)}$, and $\pi_{\left(j, G_{i}\right)}^{l_{i_{k}}} \geq 0$ denotes the price user $\left(j, G_{i}\right)$ is willing to pay at link $l_{j_{k}}$ of his route $\mathcal{R}_{\left(j, G_{i}\right)}$.

Outcome function: For any $m \in \mathcal{M}$, the outcome function is

A. Kakhbod is with the Department of Electrical and System Engineering University of Pennsylvania, Philadelphia (e-mail: akakhbod@seas.upenn.edu).

D. Teneketzis is with the Department of Electrical Engineering and Computer Science, University of Michigan (e-mail: teneket@eecs.umich.edu).

Digital Object Identifier 10.1109/JSAC.2013.130717. defined as follows:

$$
\begin{aligned}
f(m) & =\left(\left(x_{\left(i, G_{1}\right)}, t_{\left(i, G_{1}\right)}\right), \cdots,\left(x_{\left(k, G_{N}\right)}, t_{\left(k, G_{N}\right)}\right)\right) \\
t_{\left(j, G_{i}\right)} & =\sum_{l \in \mathcal{R}_{\left(j, G_{i}\right)}} t_{\left(j, G_{i}\right)}^{l},
\end{aligned}
$$

where $t_{\left(j, G_{i}\right)}^{l}$ is tax paid by user $\left(j, G_{i}\right)$ for using link $l$. The form of $t_{\left(j, G_{i}\right)}^{l}$ is the same as the tax function defined in [1] excluding the terms denoted by $\Xi_{\left(j, G_{i}\right)}^{l}$. For example, in Part DI where $\left|G_{i}{ }^{\max }(l)\right| \geq 2$, the tax function in Eq. (14) of [1] is modified as follows:

Let the label of $\left(j, G_{i}\right)$ in $G_{i}^{\max }(l)$ be $\left(k, G_{i}^{\max }(l)\right)$. Then:

(i). If $\left(j, G_{i}\right) \in G_{i}^{\max }(l)$,

$$
\begin{aligned}
t_{\left(j, G_{i}\right)}^{l} & =\pi_{\left(k+1, G_{i} \max (l)\right)} x_{\left(j, G_{i}\right)} \\
& +\frac{\left(P_{G_{i} \max (l)}-P_{-G_{i} \max (l)}-\eta_{+}^{l}\right)^{2}}{\left|G_{i}{ }^{\max }(l)\right|} \\
& -2 \frac{P_{-G_{i} \max (l)}}{\left|G_{i}^{\max }(l)\right|}\left(P_{G_{i} \max (l)}\right. \\
& \left.-P_{-G_{i}{ }^{\max }(l)}\right)\left[\frac{\mathcal{E}_{-G_{i} \max (l)}+x_{\left(j, G_{i}\right)}}{\gamma}\right] \\
& +\frac{\Gamma_{G_{i}}^{l}}{\left|G_{i}^{\max }(l)\right|},
\end{aligned}
$$

where

$$
\eta_{+}^{l}=\max \left\{0, \frac{\sum_{G_{i} \in Q_{l}} x_{G_{i}}(l)-c_{l}}{\hat{\gamma}}\right\},
$$

$c_{l}$ is the capacity of link $l, \mathcal{E}_{-G_{i}}{ }^{\max (l)}, P_{G_{i} \max (l)}, P_{-G_{i}}{ }^{\max }(l)$ and $\Gamma_{G_{i}}^{l}$ are defined by equations (18)-(21) in [1], and $\gamma, \hat{\gamma}$ are positive constants.

(ii). If $\left(j, G_{i}\right) \notin G_{i}^{\max }(l)$ then

$$
t_{\left(j, G_{i}\right)}^{l}=\pi_{\left(k+1, G_{i}{ }^{\max }(l)\right)}\left(\mathcal{E}_{-G_{i}{ }^{\max }(l)}+x_{G_{i}}(l)\right) .
$$

This completes the specification of the mechanism.

Based on the above specification, the proof of Lemma 3 in [1] is updated as follows.

Proof of Lemma 3 in [1]: Let $m^{*}$ be a NE of the game induced by the mechanism. Consider $G_{i} \in Q_{l}$, and $\left(k, G_{i}^{\max }(l)\right) \in G_{i}^{\max }(l)$. Since user $\left(k, G_{i}^{\max }(l)\right)$ does not control $\Gamma_{G_{i}}^{l}$ (as in [1], page 2101), $\frac{\partial \Gamma_{\left(k, G_{i}^{\max }(l)\right)}}{\partial \pi_{\left(k, G_{i}^{\max }(l)\right)}}=0$. By following the same steps as in equations (32-40) of [1], we 
obtain:

$$
\begin{aligned}
\left.\frac{\partial t_{\left(k, G_{i}^{\max }(l)\right)}^{l}}{\left.\partial \pi_{\left(k, G_{i}{ }^{\max }(l)\right.}\right)}\right|_{\boldsymbol{m}=\boldsymbol{m}^{*}} \\
=\frac{-2}{\left|G_{i}{ }^{\max }(l)\right|} P_{-G_{i}{ }^{\max }(l)}^{*}\left(\frac{\mathcal{E}_{-G_{i}{ }^{\max }(l)}^{*}+x_{G_{i}}^{*}(l)}{\gamma}\right) \\
\quad+\frac{2}{\left|G_{i}{ }^{\max }(l)\right|}\left(P_{G_{i} \max (l)}^{*}-P_{-G_{i} \max (l)}^{*}-\eta_{+}^{* l}\right) \\
=0 .
\end{aligned}
$$

Furthermore (as in Eq. (36) in [1]) we note that $\sum_{G_{i} \in Q_{l}} P_{G_{i} \max (l)}=\sum_{G_{i} \in Q_{l}} P_{-G_{i} \max (l)}$. Thus, summing (3) over all $G_{i} \in Q_{l}$, and $\left(k, G_{i}^{\max }(l)\right) \in G_{i}{ }^{\max }(l)$, we get

$$
\begin{aligned}
& \sum_{G_{i} \in Q_{l}} \sum_{\left(k, G_{i}^{\max }(l)\right) \in G_{i}{ }^{\max }(l)} \frac{\partial t_{\left(k, G_{i}^{\max }(l)\right)}^{l}}{\left.\partial \pi_{\left(k, G_{i}\right.} \max _{(l)}\right)} \\
& =-\left|Q_{l}\right| \eta_{+}^{* l}-\sum_{G_{i} \in Q_{l}} P_{-G_{i}{ }^{\max (l)}}^{*}\left(\frac{\mathcal{E}_{-G_{i}{ }^{\max }(l)}+x_{G_{i}}^{*}(l)}{\gamma}\right) \\
& =0 .
\end{aligned}
$$

Suppose $\sum_{G_{i} \in Q_{l}} x_{G_{i}}^{*}(l)-c_{l}>0$. Then we must have $\eta_{+}^{*}>$ 0 and $\sum_{G_{i} \in Q_{l}} P_{-G_{i} \max (l)}^{*}\left(\frac{\mathcal{E}_{-G_{i} \max (l)}^{*}+x_{G_{i}}^{*}(l)}{\gamma}\right) \geq 0$. But this contradicts (4). Therefore, we must have

$$
\sum_{G_{i} \in Q_{l}} x_{G_{i}}^{*}(l) \leq c_{l} .
$$

Inequality (5) implies,

$$
\eta_{+}^{* l}=0 .
$$

Combining (6) along with (4) we obtain

$$
\sum_{G_{i} \in Q_{l}} P_{-G_{i} \max (l)}^{*}\left(\frac{\mathcal{E}_{-G_{i} \max (l)}^{*}+x_{G_{i}}^{*}(l)}{\gamma}\right)=0 .
$$

Moreover, combining (6) and (7) we obtain

$$
P_{-G_{i} \max (l)}^{*}\left(\frac{\mathcal{E}_{-G_{i} \max (l)}^{*}+x_{G_{i}}^{*}(l)}{\gamma}\right)=0 .
$$

for any $G_{i} \in Q_{l}$. Using (8) and (6) in (3) we obtain

$$
P_{-G_{i} \max (l)}^{*}=P_{G_{i} \max (l)}^{*} \text {. }
$$

Since (9) is true for all $G_{i} \in Q_{l}$, it implies

$$
P_{-G_{i} \max (l)}^{*}=P_{G_{i} \max (l)}^{*}=P_{G_{j} \max (l)}^{*}=: P_{G^{\max (l)}}^{*}, \forall G_{i} \in Q_{l}
$$

and along with (8) it implies

$$
P_{G^{\max }(l)}^{*}\left(\mathcal{E}_{-G_{i} \max (l)}^{*}+x_{G_{i}}^{*}(l)\right)=0 .
$$

Furthermore, because of

$$
\frac{\partial \Gamma_{\left(k, G_{i}^{\max }(l)\right)}}{\partial x_{G_{i}}(l)}=0
$$

(as in [1], page 2101), and (6), (8), (10), Eqs. (1) and (2) give

$$
\left.\frac{\partial t_{\left(k, G_{i}^{\max }(l)\right)}^{l}}{\partial x_{G_{i}}(l)}\right|_{\boldsymbol{m}=\boldsymbol{m}^{*}}=\pi_{\left(k+1, G_{i} \max (l)\right)}^{*} .
$$

\section{Properties of THE MeChanism}

Existence of Nash equilibria (NE): The proof of existence of $\mathrm{NE}$ of the game induced by the mechanism is the same as in Theorem 1 of [1].

Feasibility of allocations at NE: Because of the specification of the mechanism and Eq. (6), the allocations corresponding to all NE are in the feasible set.

Budget Balance at any NE: Budget balance at any NE follows by Lemma 4 of [1].

Individual Rationality: Individual rationality follows by Theorem 5 of [1].

Nash implementation: Nash implementation follows by Theorem 6 of [1].

\section{REFERENCES}

[1] A. Kakhbod and D. Teneketzis, "An efficent game form for multi-rate multicast service provisioning", IEEE J. on Selected Areas in Communications: Special Issue on the Economics of Communication Networks and Systems, vol. 30, no. 11, pp. 2093-2104, December 2012. 